ARTICLE

https://doi.org/10.1038/s41467-021-21947-1

\title{
Dual $\mathrm{Ni}$ /photoredox-catalyzed asymmetric cross-coupling to access chiral benzylic boronic esters
}

Purui Zheng ${ }^{1,2}$, Pan Zhou', Dong Wang ${ }^{1}$, Wenhao Xu1, Hepan Wang ${ }^{1} \&$ Tao XU (iD ${ }^{1 凶}$

The flourishing $\mathrm{Ni}$ /photoredox-catalyzed asymmetric couplings typically rely on redoxneutral reactions. In this work, we report a reductive cross-coupling of aryl iodides and $\alpha$ chloroboranes under a dual catalytic regime to further enrich the metallaphotoredox chemistry. This approach proceeds under mild conditions (visible light, ambient temperature, no strong base) to access the versatile benzylic boronic esters with good functional group tolerance and excellent enantioselectivities.

\footnotetext{
${ }^{1}$ Shanghai Key Laboratory of Chemical Assessment and Sustainability, School of Chemical Science and Engineering, Tongji University, Shanghai, People's Republic of China. ${ }^{2}$ Department of Polymeric Materials, School of Materials Science and Engineering, Tongji University, Shanghai, People's Republic of China.

凶email: taoxu@tongji.edu.cn
} 
$\mathrm{T}$ he merger of photoredox catalysis with transition metal catalysts, named as "metallaphotoredox", has become a popular strategy to construct $\mathrm{C}-\mathrm{C}$ bonds in the past decade $^{1}$. This dual catalytic platform has led to many new discoveries, which were unfeasible or difficult to achieve with a single catalytic system ${ }^{2-6}$. Such cooperative regime, especially with nickel, provides a novel catalytic mode in asymmetric crosscouplings under exceptionally mild conditions and avoids the use of air-sensitive organometallic reagents or stoichiometric metal reductants (Fig. 1a) ${ }^{7,8}$. Currently, dual Ni/photoredoxcatalyzed asymmetric couplings typically involve redox-neutral methods, such as Suzuki-Miyaura reactions, but few reductive cross-couplings have been reported ${ }^{9-15}$. We speculated that the application of dual catalytic system in asymmetric reductive cross-couplings could enable the use of various electrophiles and increase the range of accessible reaction types ${ }^{16-18}$. In addition, more ligand selections are likely to achieve high enantioselectivity in the absence of stoichiometric metal reductants because of circumventing the possible coordination between the metal reductant and the ligand.

However, the challenges also remain. For instance, rigorous coordinative control over several reduction cycles is needed to avoid homo-couplings or other side products. Furthermore, because of the high tendency to protodehalogenation of both halide components in the presence of reducing reagents (amines, Hantzsch ester (HEH)), the efficiency is limited. Based on these challenges, the application of this strategy upon the enantioselective reductive cross-couplings is still very less to date.
As a privileged structural class, chiral benzylic boronic esters are important precursors in organic synthesis due to their versatilities on the stereospecific conversion to many kinds of bonds ${ }^{19}$. In addition to the ease of handling and high air- and moisture-stability, many methods have therefore been devised to access enantioenriched benzylic boronic esters, such as hydroboration of alkenes ${ }^{20-23}$ or alkynes ${ }^{24}$, and others ${ }^{25-28}$. Meanwhile, asymmetric cross-couplings are also very popular (Fig. 1b). A palladium-catalyzed asymmetric coupling of bis(pinacolato) diboron $\left(\mathrm{B}_{2} \mathrm{Pin}_{2}\right)$ with aryl halides, mainly bearing electrondonating groups, was reported by the Morken group ${ }^{29}$. A nickeland a copper-catalyzed enantioselective borylation of secondary benzylic chlorides with $\mathrm{B}_{2} \mathrm{Pin}_{2}$, were published by the $\mathrm{Fu}^{30}$ and Ito $^{31}$ group, respectively. However, in these cases, the enantiomeric excess $(e e)$ values of the products were generally not ideal $(<87 \%)$. Although several racemic couplings under reductive conditions have been developed ${ }^{32-36}$, to the best of our knowledge, asymmetric reductive cross-coupling with the dual catalytic system constitutes a potentially powerful yet unreported route to construct chiral benzylic boronic esters. Herein, we report a Ni/ photoredox-catalyzed asymmetric coupling with broad substrate scopes and excellent enantioselectivities (basically above $90 \% e e$ ) by using a simple, commercially available ligand (Fig. 1c).

\section{Results}

Reaction conditions development. We commenced our investigation of this asymmetric coupling with the reaction between 4-

a Catalytic Modes on Asymmetric Cross-Coupling

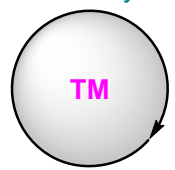

well-established; many types. organometallic reagents or stoichiometric metal reductants

v.s.

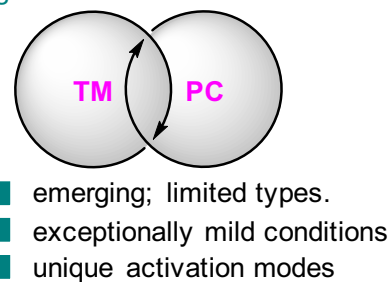

b The Routes to Access Chiral Benzylic Boronic Esters via Coupling

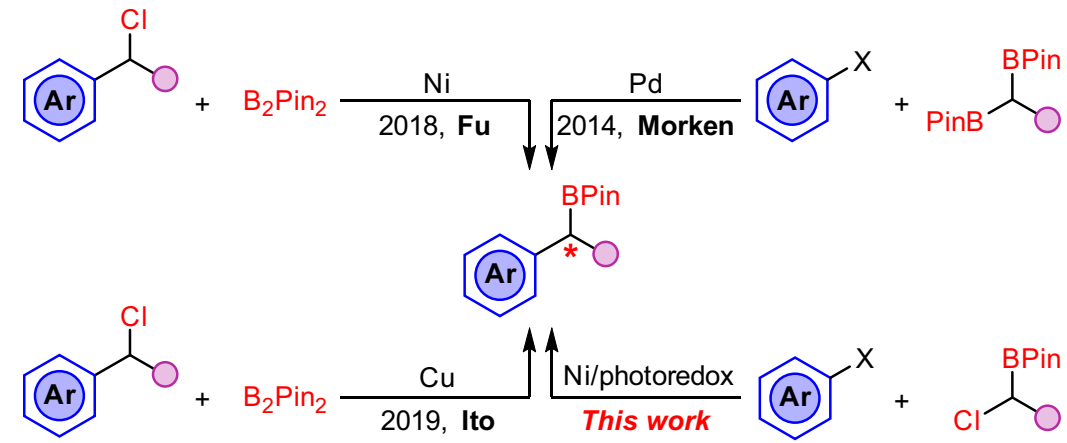

C Ni/Photoredox-Catalyzed Asymmetric Process

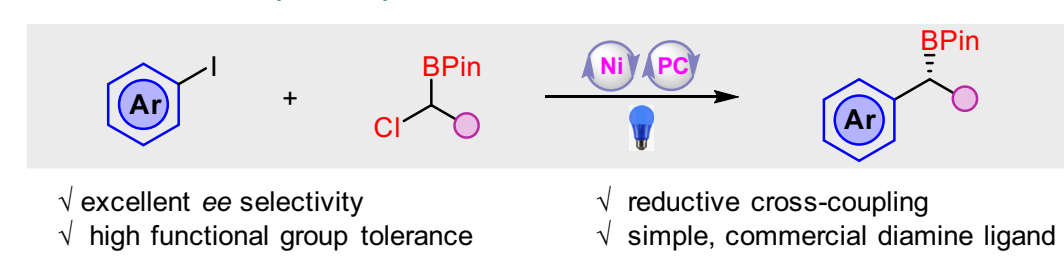

Fig. 1 TM-catalyzed asymmetric cross-couplings. a Catalytic modes on asymmetric cross-coupling. $\mathbf{b}$ The routes to access chiral benzylic boronic esters via coupling. c Ni/photoredox-catalyzed asymmetric process. (TM: transition metal; PC: photoredox catalyst). 


\section{Table 1 Optimization of reaction conditions.}

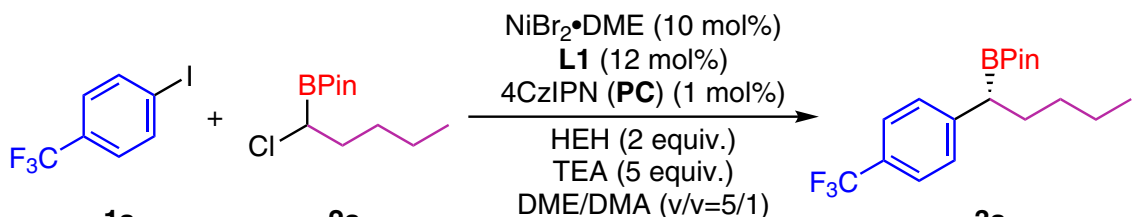

$1 \mathbf{a}$

$2 a$ Blue LEDs $(30 \mathrm{~W})$
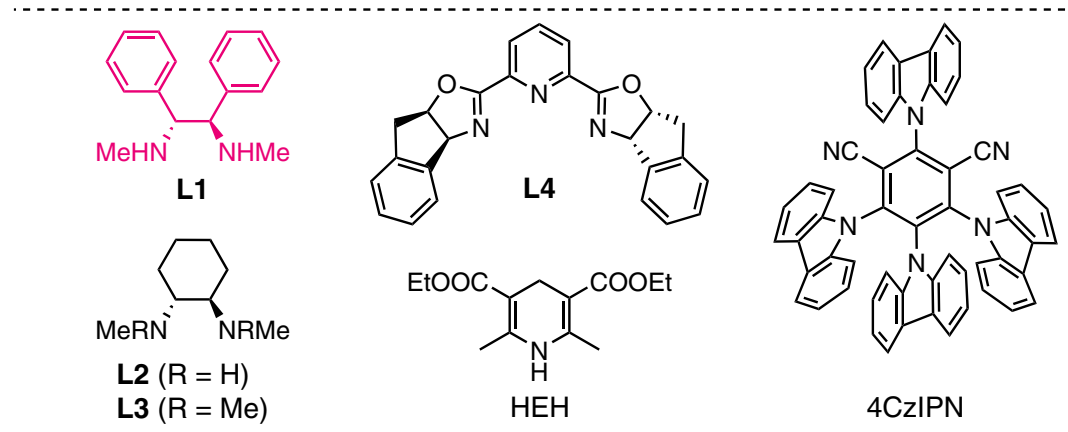

\begin{tabular}{|c|c|}
\hline Entry ${ }^{a}$ & Changes \\
\hline 1 & No change \\
\hline 2 & Using $\mathrm{Ni}(\operatorname{cod})_{2}$ \\
\hline 3 & Using $\mathrm{Nil}_{2}$ \\
\hline 4 & L2 instead of L1 \\
\hline 5 & L3 instead of L1 \\
\hline 6 & L4 instead of L1 \\
\hline 7 & {$\left[\operatorname{lr}\left(\mathrm{dFCF}_{3} \mathrm{ppy}\right) \mathrm{dtbbpy}\right] \mathrm{PF}_{6}$ as $\mathrm{PC}$} \\
\hline 8 & $\mathrm{Ru}(\mathrm{bpy})_{3} \mathrm{Cl}_{2} 6 \mathrm{H}_{2} \mathrm{O}$ as PC \\
\hline 9 & DME as solvent \\
\hline 10 & DMA as solvent \\
\hline 11 & $\mathrm{No} t_{3} \mathrm{~N}$ \\
\hline 12 & $\mathrm{Li}_{2} \mathrm{CO}_{3}$ instead of TEA \\
\hline 13 & No HEH \\
\hline 14 & No 4CzIPN \\
\hline 15 & No $\mathrm{Ni}$ or no light \\
\hline
\end{tabular}

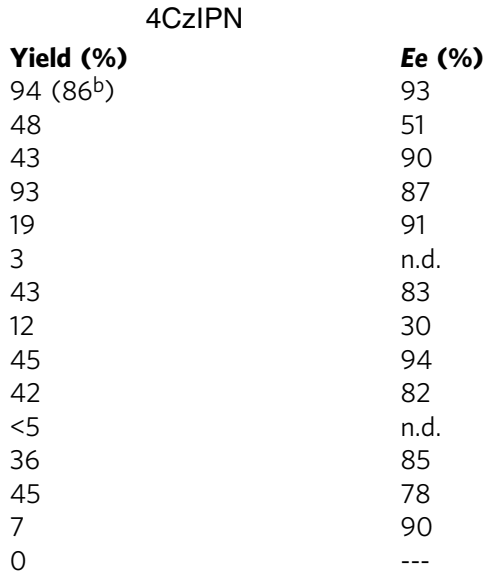

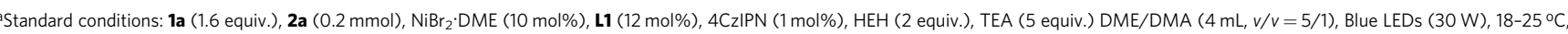
$8-10 \mathrm{~h}$. Yields were determined by $\mathrm{GC}$ with ${ }^{n}$ dodecane as an internal standard. The ee values were determined by HPLC.

bIsolated yield was given in the parenthesis.

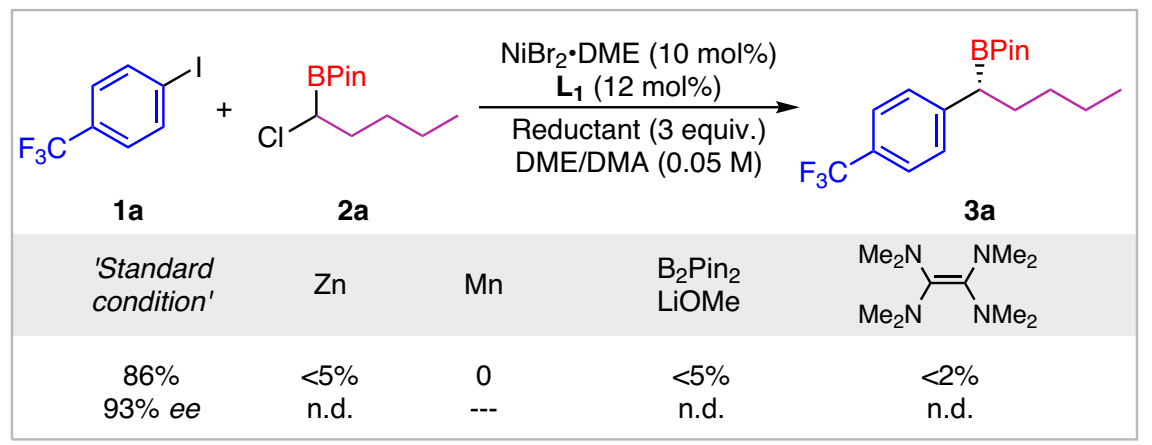

Fig. 2 The reaction with other reductants. Comparison reaction with other reductive conditions.

iodobenzotrifluroride (1a) and a-chloroborane (2a) with dual $\mathrm{Ni}$ / photoredox catalysts ${ }^{37}$. Systematic evaluation of all the reaction parameters indicated that the transformation proceeded smoothly under the conditions listed in entry 1 , Table 1 , to give the product $3 \mathbf{a}$ in $94 \%$ yield with $93 \%$ ee (Table 1 , entry 1 ). Other Ni catalysts gave lower conversions (entries 2-3). The use of other ligands
(L2, L3), especially the bis(oxazoline) ligand $\mathbf{L 4}$, which is particularly common in cross-couplings ${ }^{30}$, resulted in diminished outcomes (entry 4-6). Examination of other photocatalysts or solvents identified a rather significant decrease in yields and/or enantioselectivities. (entries 7-10, see supporting information). Although the exact reasons remain elusive, the mixed solvent 
Table 2 Scope of Ni/photoredox-catalyzed coupling of aryl iodides with $\alpha$-chloroboranes ${ }^{\mathrm{a}}$.

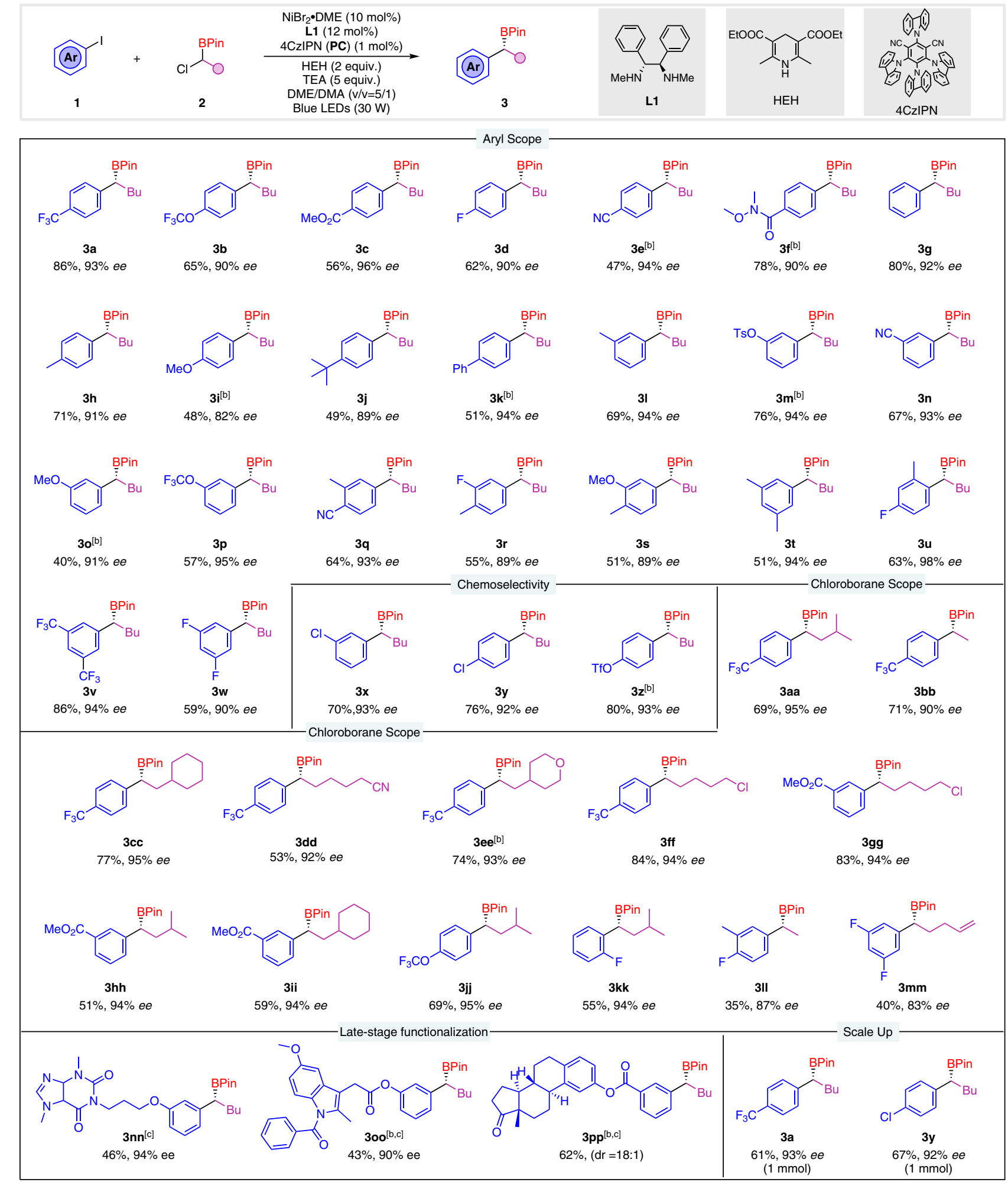

aReaction conditions: 1 ( 1.6 equiv.), 2 ( $0.2 \mathrm{mmol}), \mathrm{NiBr} 2 \cdot \mathrm{DME}(10 \mathrm{~mol} \%), \mathbf{L 1}(12 \mathrm{~mol} \%), 4 \mathrm{CzIPN}(1 \mathrm{~mol} \%), \mathrm{HEH}\left(2\right.$ equiv.), TEA ( 5 equiv.), DME/DMA (4 mL, v/v = 5/1), Blue LEDs (30 W), $18-25^{\circ} \mathrm{C}$, 8-10 h. Isolated yields were given. The ee values were determined of the corresponding alcohol after oxidation by HPLC. 

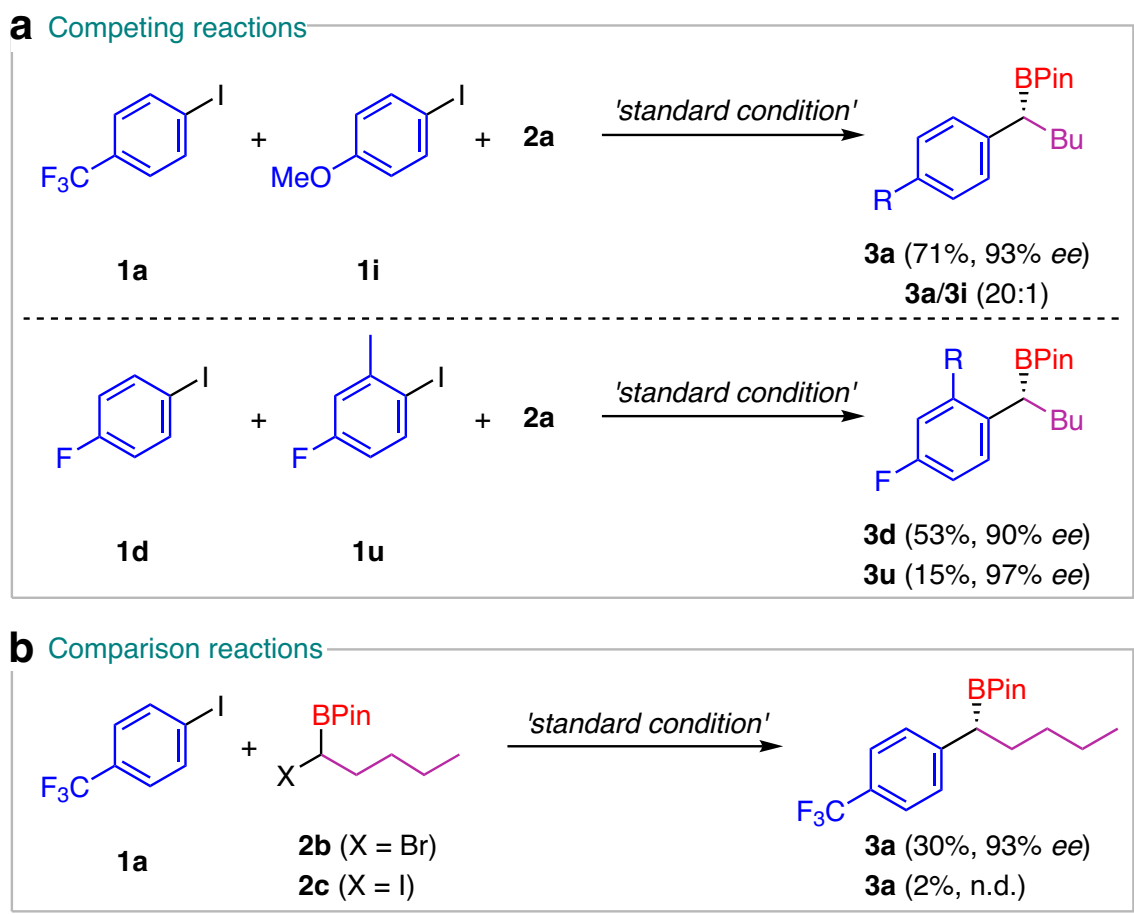

Fig. 3 Competing and comparison reactions. a The reaction with aryl halides with different properties. $\mathbf{b}$ Test on the $\alpha$-bromo- or $\alpha$-iodoborane.

system possibly increased the dielectric constant of the reaction medium $^{30,38}$, thus improving the catalytic efficiency and suppressing the protodehalogenation product. A trace amount of product was detected in the absence of $\mathrm{Et}_{3} \mathrm{~N}$ and a low yield was obtained with $\mathrm{Li}_{2} \mathrm{CO}_{3}$ (entries 11-12). Considering $\mathrm{Et}_{3} \mathrm{~N}$ can act as a reductant in photoredox-catalyzed reactions, it is reasonable that the reaction still worked without $\mathrm{HEH}$, albeit with lower efficiency (entry 13). The reaction can proceed for one or fewer catalytic cycles without the photocatalyst, which may suggest a mechanistic scenario more complex than previous proposition (entry 14). Control reactions performed in the absence of $\mathrm{Ni}$ catalyst or light resulted in no detectable product formation, confirming the essential role of each of these components in the dual catalytic process (entry 15).

In addition, perhaps most notable, the comparison reactions with other common reductive systems were tested to identify the difference and powerful efficiency of this Ni/photoredoxcatalyzed coupling (Fig. 2). The results showed that whether the metal reductants $\mathrm{Zn} / \mathrm{Mn}$ or the organic reagents $\mathrm{B}_{2} \mathrm{Pin}_{2}$ and tetrakis(dimethylamino)ethylene (TDAE) gave only traces of the product or no product, thus further strengthening the high superiority of the dual catalytic method on this $\mathrm{C}-\mathrm{Cl}$ bond transformation.

Substrate scope. Having developed the enantioselective catalytic reaction of this cross-coupling, we sought to investigate the generality and utility of this dual $\mathrm{Ni}$ /photoredox-catalyzed method (Some products were found to be prone to decompose in column, thus isolated yields after oxidation were given. See Table 2). We firstly tested various aryl iodides with either electron-withdrawing or electron-donating groups (the reaction with $p-\mathrm{CF}_{3}-\mathrm{C}_{6} \mathrm{H}_{4} \mathrm{Br}$ gave the product $3 \mathrm{a}$ in $21 \%$ yield with $91 \% e e$ under the standard conditions). The excellent enantioselectivities and satisfactory yields were generally given whether the substituents on the para-, meta-, or ortho- positions, in an effort to showcase the mild nature of this transformation. The electronpoor substrates obviously facilitated the couplings, as demonstrated with compounds $\mathbf{3 a}-\mathbf{3 f}$. The reactions with strong electron-rich aryl iodides (3i) led to a decreased but still acceptable results, thus serving to highlight the complementary and discrepant nature of this method compared to the established method with palladium where the two-electron transmetalation regime was involved ${ }^{29}$. The chemoselectivity with different electrophiles was studied under the current conditions. The exclusive selection on aryl iodide was observed in the presence of aryl chloride or triflate group $(\mathbf{3 x}, \mathbf{3 y}, \mathbf{3 z})$ while a mixture was obtained with 1-bromo-4-iodobenzene as a substrate, possibly due to the existing reactivity of aryl bromides. It is worth mentioning that, this method can afford high enantioselectivities not only for the bulky or ortho-substituted aryl substrates as previous reports ${ }^{29-31}$, but also for the less sterically hindered components (such as $\mathbf{3 g}, \mathbf{3 h}$ ), thus expanding its application in organic synthesis.

The scope of $\alpha$-chloroborane was then explored in the dual catalytic system. Overall, a wide range of the chloroboronic esters could be smoothly transferred to the corresponding products with very high ee values. For the complex molecules (3nn-3pp), this Ni/photoredox-catalyzed coupling was also suitable, thus demonstrating the potential of this methodology in the late-stage functionalization. The reaction also exhibited comparable efficiency on a larger scale, as the products $(\mathbf{3 a}, \mathbf{3 y})$ were isolated in good yields and undiminished ee on a $1 \mathrm{mmol}$ scale. In addition, Table 2 also highlighted the robustness on functional group compatibility of this approach. The trifluoromethyl (3a, 3v), trifluoromethoxy (3b, 3p), ester (3c, 3hh, 3ii), amide (3f), nitrile (3e, 3n, 3dd), aryl halides (3d, 3w, 3x), triflate $(\mathbf{3 z})$, phenol $(\mathbf{3 m})$, ether (3i, 3ee), alkyl chloride (3ff, $\mathbf{3 g g})$, alkenyl (3mm) groups were all compatible under the optimized conditions.

Competing and comparison reactions. To clarify the features on this dual catalytic system, competing reactions were conducted. Although both electron-rich and electron-poor aryl iodides can participate in this transformation, the substrate with an electron- 


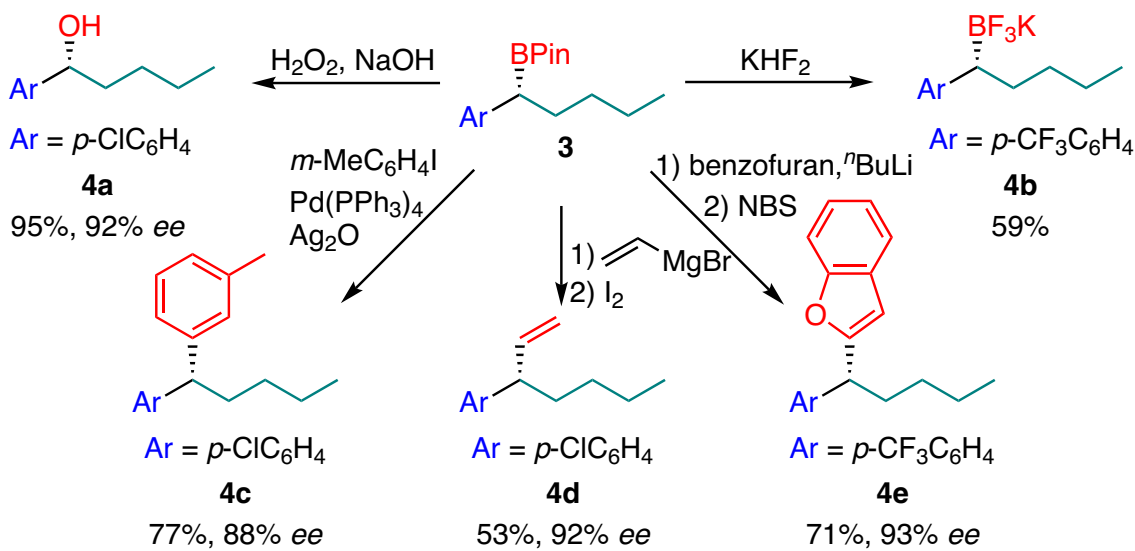

Fig. 4 Further application of the products. Derivatization of the chiral benzylic boronic esters.

a Cyclization reactions

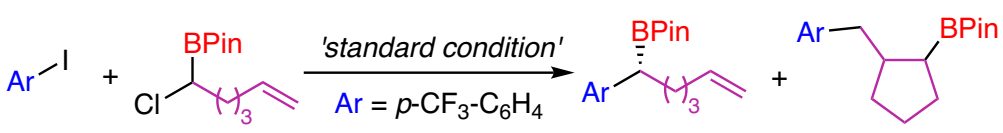

$1 \mathbf{a}$

2d

\section{$3 q q$}

$5 a$

$5 \% \quad 52 \%($ d.r. $=1.5: 1)$

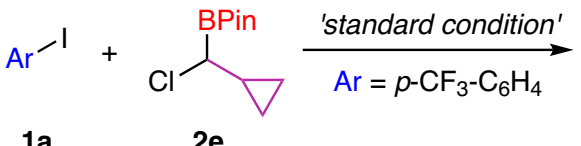<smiles>FC(F)(F)c1ccc(CC/C=C/[B]c2ccccc2)cc1</smiles>

$5 b$

$78 \%(E / Z>20: 1)$

b Reactions with radical probe

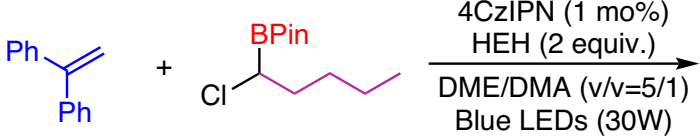

6

$2 a$<smiles>CCCCC([B]c1ccccc1)CC(c1ccccc1)c1ccccc1</smiles>

7

trace

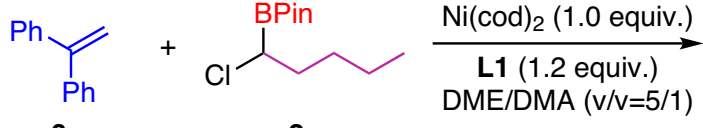

6

$2 a$<smiles>CCCCC([B]c1ccccc1)CC(c1ccccc1)c1ccccc1</smiles>

7

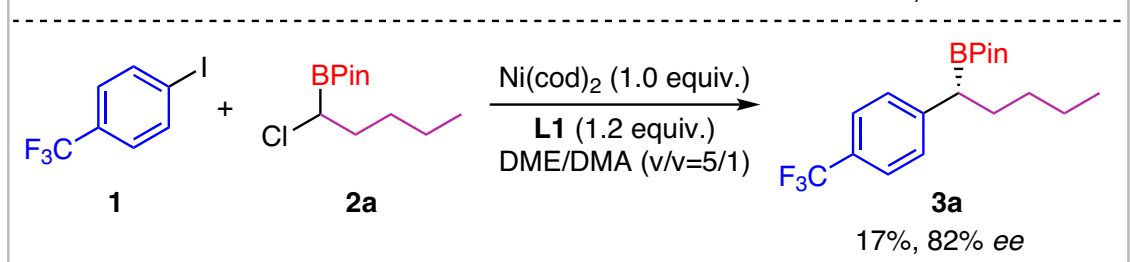

Fig. 5 Mechanistic studies. a Cyclization reactions. b Reactions with radical probe.

withdrawing group favored when both are present, as evidenced by isolation of product $\mathbf{3 a}$ in Fig. 3a. Steric effect was also investigated. When $\mathbf{1 d}$ and $\mathbf{1 u}$ were used simultaneously, the less sterically hindered component gave the main product $\mathbf{3 d}$.

a-Bromo- and $\alpha$-iodoboranes, which are often used in $\mathrm{Ni}$ catalyzed cross-couplings ${ }^{34,39}$, were also tested (Fig. 3b). Surprisingly, when a-bromoborane was employed, the target product was isolated in only $30 \%$ yield with a similar enantioselectivity. A trace amount of product was observed in GC when the more active $\alpha$-iodoborane was used. The protodehalogenation product was the main product in these two reactions. These results show the importance of concerted rates of reductive processes in this dual catalytic system and the difference from the single catalytic regime.

Further applications. One advantage of this method is that the resulting molecules contain a versatile boron group, which is convenient for further functionalization to construct many other chiral 

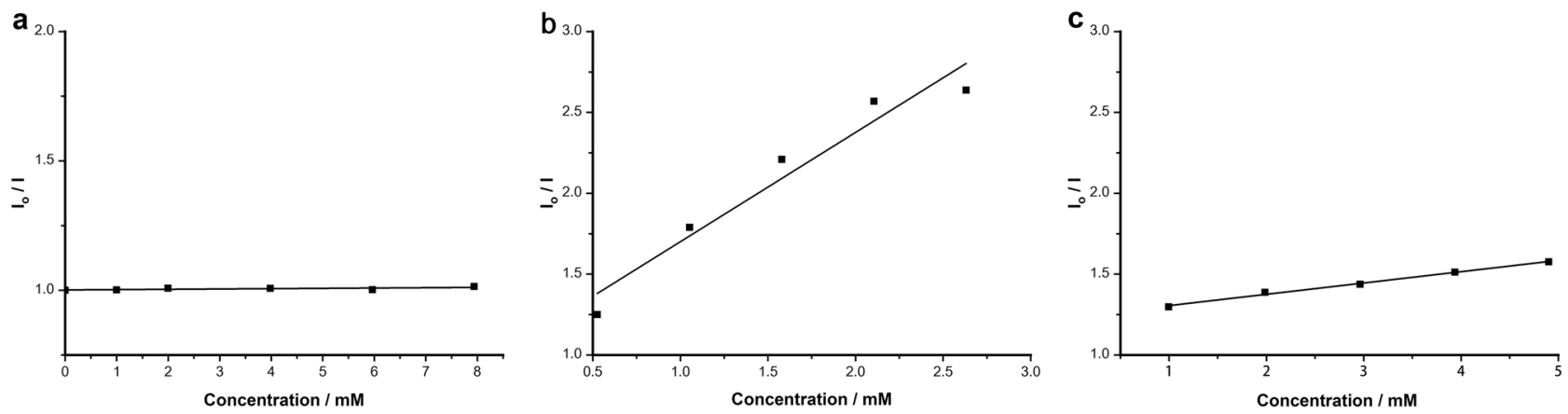

Fig. 6 Stern-Volmer quenching experiments. a With substrate 2a. b With $\mathrm{HEH}$. c With $\mathrm{Et}_{3} \mathrm{~N}$.

bonds. We demonstrated this in a series of examples in Fig. 4. For example, the boranes can be oxidized to chiral alcohol (4a) or transformed to the potassium trifluoroborate salt $(4 \mathbf{b})$ in good yields. The chiral 1,1-diarylalkanes, which are widely useful but not easily accessible, can be conveniently obtained from these products with a Pd-catalyzed coupling reaction (4c). Meanwhile, some other $\mathrm{C}_{\mathrm{sp} 3}-\mathrm{C}_{\mathrm{sp} 2}$ bonds were readily formed to introduce alkenyl or heterocycles according to the reported methods $(\mathbf{4 d}, \mathbf{4 e})^{40,41}$.

Mechanistic studies. To give some insights into the mechanism of the coupling, some experiments were performed (Fig. 5). The substrate $\mathbf{2 d}$ with an alkene chain was investigated. The cyclization product 5 a was isolated in $52 \%$ yield and direct crosscoupling 3qq was only detected in 5\% GC yield, indicating the production of the radical intermediate. The same conclusion is also deduced from the reaction with radical clock substrate $\mathbf{2 e}$ (Fig. 5a). Although it was reported that an alkyl halide can be reduced to a radical species via a single-electron reduction by photocatalysts $^{42}$, herein, the luminescence quenching experiments indicated that $\mathrm{HEH}$ and $\mathrm{Et}_{3} \mathrm{~N}$ are more likely to quench the excited state of $4 \mathrm{CzIPN}$ (Fig. 6). The reactions with a radical probe also proved this point (Fig. $5 b$ ). In the absence of $\mathrm{Ni} / \mathbf{L} \mathbf{1}$, the a-chloroborane can not react with 1,1-diphenylethylene 6 to give any radical-trapped product 7 . However, in the stoichiometric studies with $\mathrm{Ni}(\operatorname{cod})_{2} / \mathbf{L 1}$, product 7 can be obtained in $30 \%$ yield with no enantioselectivity. Hence, the free alkyl radical should be generated from $\alpha$-chloroborane by the reduction of $\mathrm{Ni}$ species rather than $4 \mathrm{CzIPN}^{43-46}$.

Based on these experiments, a proposed mechanism is shown in Fig. 7. Light excitation of photocatalyst 4CzIPN generates the photoexcited [4CzIPN] ${ }^{*}$, which is reduced by $\mathrm{HEH}$ or $\mathrm{Et}_{3} \mathrm{~N}$ to the reduced ground-state photocatalyst [4CzIPN]--. Then, combination with the nickel cycle, it is responsible to produce a boron-stabilized radical I. Meanwhile, the oxidative addition of aryl iodide to the ligated $\mathrm{Ni}(0)$ II forms a $\mathrm{Ni}$ (II) species III. Then oxidation by radical I accesses the high-valent $\mathrm{Ni}$ (III) intermediate IV, and subsequent reductive elimination affords the product and releases $\mathrm{Ni}(\mathrm{I})$ species $\mathbf{V}$. Reduction of $\mathbf{V}$ would regenerate the $\mathrm{Ni}(0)$ and $4 \mathrm{CzIPN}$ catalyst, closing the dual catalytic cycle. However, at this moment, we can not exculde another catalytic cycle in which the alkyl radical is trapped by $\mathrm{Ni}$ (0), then the alkyl $\mathrm{Ni}(\mathrm{I})$ species is oxidated by aryl hailde ${ }^{47,48}$.

\section{Discussion}

In summary, herein a dual $\mathrm{Ni}$ /photoredox-catalyzed asymmetric reductive cross-coupling of aryl iodides with $\alpha$-chloroboranes was developed. The mild conditions enable the process to give excellent enantioselectivities and accommodate a broad palette of architectures with many functional groups. Mechanistic studies suggest the alkyl radical is probably generated via reduction by

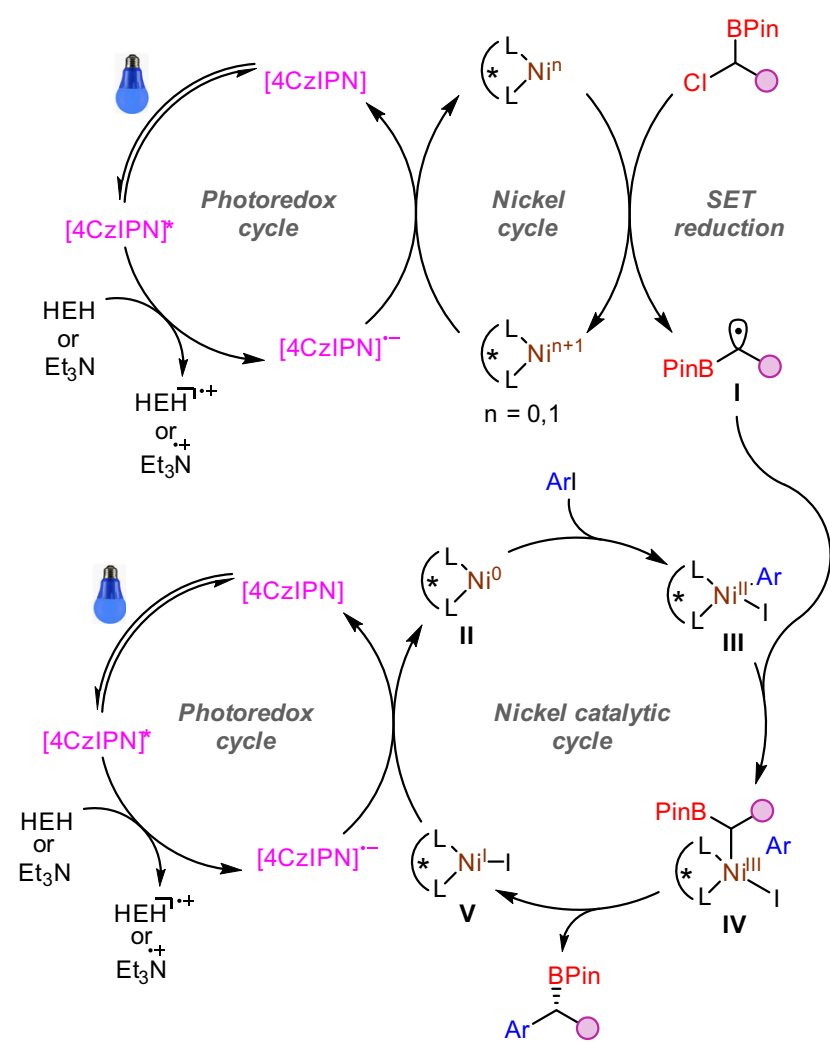

Fig. 7 Proposed mechanism. A proposed catalytic cycle for the dual catalytic coupling.

nickel. The resulting chiral benzylic boronic esters, which have important versatilities in organic synthesis demonstrated the high potential of this transformation.

\section{Methods}

General procedure for the dual catalytic coupling. In a $\mathrm{N}_{2}$-filled glovebox, an oven-dried $10-\mathrm{mL}$ Schlenk tube containing a Teflon stir bar was charged with 4CzIPN (0.002 mmol), NiBr 2 .DME (0.02 mmol), L1 (0.024 mmol), HEH (0.4 mmol), TEA $(1.0 \mathrm{mmol})$. Then the tube was sealed with a septum and taken out. DME/DMA $(v / v=5 / 1,4 \mathrm{~mL})$ were added via syringe under $\mathrm{N}_{2}$ atmosphere. After stirring for $30 \mathrm{~min}$ at room temperature, aryl halide $(0.32 \mathrm{mmol})$ and $\alpha-$

chloroborane $(0.2 \mathrm{mmol})$ were added. The reaction mixture was stirred and irradiated under blue light $(\lambda=450-455 \mathrm{~nm})$ for $8-10 \mathrm{~h}$, while the temperature was controlled at $18-25^{\circ} \mathrm{C}$. Upon completed, the mixture was diluted with EtOAc and quenched with water. The aqueous solution was extracted with EtOAc three times. The combined organic layers were dried, concentrated and purified by flash column chromatography using PE/EtOAc as the eluent to afford the coupling product. The $e e$ value was determined by high-performance liquid chromatography analysis using the corresponding alcohol after oxidation of the product. 


\section{Data availability}

The authors declare that the data supporting the finding of this study are available within the paper and its Supplementary Information file. The experimental procedures and characterization of all new compounds are provided in Supplementary Information.

Received: 23 November 2020; Accepted: 16 February 2021; Published online: 12 March 2021

\section{References}

1. Shaw, M. H., Twilton, J. \& MacMillan, D. W. C. Photoredox catalysis in organic chemistry. J. Org. Chem. 81, 6898-6926 (2016).

2. Luo, J. \& Zhang, J. Donor-acceptor fluorophores for visible-light-promoted organic synthesis: photoredox/ $\mathrm{Ni}$ dual catalytic $\mathrm{C}(\mathrm{sp} 3)-\mathrm{C}(\mathrm{sp} 2)$ cross-coupling. ACS Catal. 6, 873-877 (2016).

3. Tellis, J. C. et al. Single-electron transmetalation via photoredox/nickel dual catalysis: unlocking a new paradigm for sp3-sp2 cross-coupling. Acc. Chem. Res. 49, 1429-1439 (2016).

4. Twilton, J. et al. The merger of transition metal and photocatalysis. Nat. Rev. Chem. 1, 0052 (2017).

5. Kim, S. D., Lee, J., Kim, N.-J. \& Park, B. Y. Visible-light-mediated crosscouplings and $\mathrm{C}-\mathrm{H}$ activation via dual photoredox/transition-metal catalysis in continuous-flow processes. Asian. Asian J. Org. Chem. 8, 1578-1587 (2019).

6. Milligan, J. A., Phelan, J. P., Badir, S. O. \& Molander, G. A. Alkyl carbon-carbon bond formation by nickel/photoredox cross-coupling. Angew. Chem. Int. Ed. 58, 6152-6163 (2019).

7. Zhang, H.-H., Chen, H., Zhu, C. \& Yu, S. A review of enantioselective dual transition metal/photoredox catalysis. Sci. China Chem. 63, 637-647 (2020).

8. Lipp, A., Badir, S. O. \& Molander, G. A. Stereoinduction in metallaphotoredox catalysis. Angew. Chem. Int. Ed. 60, 1714-1729 (2021).

9. Tellis, J. C., Primer, D. N. \& Molander, G. A. Single-electron transmetalation in organoboron cross-coupling by photoredox/nickel dual catalysis. Science 345, 433-436 (2014).

10. Zuo, Z. et al. Enantioselective decarboxylative arylation of $\alpha$-amino acids via the merger of photoredox and nickel catalysis. J. Am. Chem. Soc. 138, 1832-1835 (2016).

11. Gandolfo, E., Tang, X., Raha, R. S. \& Melchiorre, P. Photochemical asymmetric nickel-catalyzed acyl cross-coupling. Angew. Chem. Int. Ed. 58 16854-16858 (2019).

12. Guan, H., Zhang, Q., Walsh, P. J. \& Mao, J. Nickel/photoredox-catalyzed asymmetric reductive cross-coupling of racemic $\alpha$-chloro esters with aryl iodides. Angew. Chem. Int. Ed. 59, 5172-5177 (2020).

13. Kainz, Q. M. et al. Asymmetric copper-catalyzed C-N cross-couplings induced by visible light. Science 351, 681-684 (2016).

14. Zhang, H.-H., Zhao, J.-J. \& Yu, S. Enantioselective allylic alkylation with 4 alkyl-1,4-dihydro-pyridines enabled by photoredox/palladium cocatalysis. $J$. Am. Chem. Soc. 140, 16914-16919 (2018).

15. Lu, F.-D. et al. Asymmetric propargylic radical cyanation enabled by dual organophotoredox and copper catalysis. J. Am. Chem. Soc. 141, 6167-6172 (2019).

16. Choi, J. \& Fu, G. C. Transition metal-catalyzed alkyl-alkyl bond formation: another dimension in cross-coupling chemistry. Science 356, eaaf7230 (2017).

17. Poremba, K. E., Dibrell, S. E. \& Reisman, S. E. Nickel-catalyzed enantioselective reductive cross-coupling reactions. ACS Catal. 10, 8237-8246 (2020).

18. Delano, T. J. \& Reisman, S. E. Enantioselective electroreducitve coupling of alkenyl and benzyl halides via nickel catalysis. ACS Catal. 9, 6751-6754 (2019).

19. Collins, B. S. L., Wilson, C. M., Myers, E. L. \& Aggarwal, V. K. Asymmetric synthesis of secondary and tertiary boronic esters. Angew. Chem. Int. Ed. 56, 11700-11733 (2017).

20. Hayashi, T., Matsumoto, Y. \& Ito, Y. Catalytic asymmetric hydroboration of styrenes. J. Am. Chem. Soc. 111, 3426-3428 (1989).

21. Nelson, H. M., Williams, B. D., Miró, J. \& Toste, F. D. Enantioselective 1,1arylborylation of alkenes: merging chiral anion phase transfer with $\mathrm{Pd}$ catalysis. J. Am. Chem. Soc. 137, 3213-3216 (2015).

22. Crudden, C. M. et al. Iterative protecting group-free cross-coupling leading to chiral multiply arylated structures. Nat. Commun. 7, 11065 (2016)

23. Chen, X., Cheng, Z. \& Lu, Z. Cobalt-catalyzed asymmetric markovnikov hydroboration of styrenes. ACS Catal. 9, 4025-4029 (2019).

24. Guo, J., Cheng, B., Shen, X. \& Lu, Z. Cobalt-catalyzed asymmetric sequential hydroboration/hydrogenation of internal alkynes. J. Am. Chem. Soc. 139, 15316-15319 (2017).

25. Lovinger, G. J. \& Morken, J. P. Ni-catalyzed enantioselective conjunctive coupling with $\mathrm{C}(\mathrm{sp} 3)$ electrophiles: a radical-ionic mechanistic dichotomy. $J$. Am. Chem. Soc. 139, 17293-17296 (2017).
26. Chen, X., Cheng, Z., Guo, J. \& Lu, Z. Asymmetric remote C-H borylation of internal alkenes via alkene isomerization. Nat. Commun. 9, 3939 (2018).

27. Tao, Z., Robb, K. A., Panger, J. L. \& Denmark, S. E. Enantioselective, lewis base-catalyzed carbosulfenylation of alkenylboronates by 1,2-boronate migration. J. Am. Chem. Soc. 140, 15621-15625 (2018).

28. Wang, W., Ding, C. \& Yin, G. Catalyst-controlled enantioselective 1,1arylboration of unactivated olefins. Nat. Catal. 3, 951-958 (2020)

29. Sun, C., Potter, B. \& Morken, J. P. A catalytic enantiotopic-group-selective suzuki reaction for the construction of chiral organoboronates. J. Am. Chem. Soc. 136, 6534-6537 (2014).

30. Wang, Z., Bachman, S., Dudnik, A. S. \& Fu, G. C. Nickel-catalyzed enantioconvergent borylation of racemic secondary benzylic electrophiles. Angew. Chem. Int. Ed. 57, 14529-14532 (2018).

31. Iwamoto, $\mathrm{H}$. et al. Copper(I)-catalyzed enantioconvergent borylation of racemic benzyl chlorides enabled by quadrant-by-quadrant structure modification of chiral bisphosphine ligands. Angew. Chem., Int. Ed. 58, 11112-11117 (2019)

32. Bera, S. \& Hu, X. Nickel-catalyzed regioselective hydroalkylation and hydroarylation of alkenyl boronic esters. Angew. Chem. Int. Ed. 58, 13854-13859 (2019)

33. Zhang, Y., Han, B. \& Zhu, S. Rapid access to highly functionalized alkyl boronates by $\mathrm{NiH}$-catalyzed remote hydroarylation of boron-containing alkenes. Angew. Chem. Int. Ed. 58, 13860-13864 (2019).

34. Sun, S.-Z. \& Martin, R. Nickel-catalyzed umpolung arylation of ambiphilic a-bromoalkyl boronic esters. Angew. Chem. Int. Ed. 57, 3622-3625 (2018).

35. Campbell, M. W., Compton, J. S., Kelly, C. B. \& Molander, G. A. Three component olefin dicarbofunctionalization enabled by nickel/photoredox dual catalysis. J. Am. Chem. Soc. 141, 20069-20078 (2019)

36. Sun, S.-Z., Duan, Y., Mega, R. S., Somerville, R. J. \& Martin, R. Site-selective 1,2-dicarbofunctionalization of vinyl boronates through dual catalysis. Angew. Chem. Int. Ed. 59, 4370-4374 (2020).

37. Xu, W., Zheng, P. \& XU, T. Dual nickel- and photoredox-catalyzed reductive cross-coupling of aryl halides with dichloromethane via a radical process. Org Lett. 22, 8643-8647 (2020).

38. Chu, C. K., Liang, Y. \& Fu, G. C. Silicon-carbon bond formation via nickelcatalyzed cross-coupling of silicon nucleophiles with unactivated secondary and tertiary alkyl electrophiles. J. Am. Chem. Soc. 138, 6404-6407 (2016).

39. Schmidt, J., Choi, J., Liu, A. T., Slusarczyk, M. \& Fu, G. C. A general, modular method for the catalytic asymmetric synthesis of alkylboronate esters. Science 354, 1265-1269 (2016).

40. Sonawane, R. P. et al. Enantioselective construction of quaternary stereogenic centers from tertiary boronic esters: methodology and applications. Angew. Chem. Int. Ed. 50, 3760-3763 (2011).

41. Bonet, A., Odachowshi, M., Leonori, D., Essafi, S. \& Aggarwal, V. K. Eanantiospecific $\mathrm{sp}^{2}-\mathrm{sp}^{3}$ coupling of secondary and tertiary boronic esters. Nat. Chem. 6, 584-589 (2014)

42. Neumann, M., Füldner, S., König, B. \& Zeitler, K. Metal-free, cooperative asymmetric organophotoredox catalysis with visible light. Angew. Chem. Int Ed. 50, 951-954 (2011)

43. Duan, Z., Li, W. \& Lei, A. Nickel-catalyzed reductive cross-coupling of aryl bromides with alkyl bromides: Et3N as the terminal reductant. Org. Lett. 18 4012-4015 (2016).

44. Paul, A., Smith, M. D. \& Vannucci, A. K. Photoredox-assisted reductive cross coupling: mechanistic insight into catalytic aryl-alkyl cross-couplings. J. Org. Chem. 82, 1996-2003 (2017).

45. Meng, Q.-Y., Wang, S. \& König, B. Carboxylation of aromatic and aliphatic bromides and triflates with $\mathrm{CO} 2$ by dual visible-light-nickel catalysis. Angew. Chem. Int. Ed. 56, 13426-13430 (2017).

46. Peng, L., Li, Z. \& Yin, G. Photochemical nickel-catalyzed reductive migratory cross-coupling of alkyl bromides with aryl bromides. Org. Lett. 20, 1880-1883 (2018).

47. Gutierrez, O., Tellis, J. C., Primer, D. N., Molander, G. A. \& Kozlowski, M. C. Nickel-catalyzed cross-coupling of photoredox-generated radicals: uncovering a general manifold for stereoconvergence in nickel-catalyzed cross-couplings. J. Am. Chem. Soc. 137, 4896-4899 (2015)

48. Yuan, M., Song, Z., Badir, S. O., Molander, G. A. \& Gutierrez, O. On the nature of $\mathrm{C}(\mathrm{sp} 3)-\mathrm{C}(\mathrm{sp} 2)$ bond formation in nickel-catalyzed tertiary radical cross-couplings: a case study of $\mathrm{Ni} /$ photoredox catalytic crosscoupling of alkyl radicals and aryl halides. J. Am. Chem. Soc. 142, 7225-7234 (2020).

\section{Acknowledgements}

We thank NFS of China (Grant No. 22071183, 21801188), Shanghai Pujiang Program, and Tongji University for financial support. 


\section{Author contributions}

T.X. designed the project. P. Zheng, P. Zhou, and D.W. performed the experiments. W.X. and H.W. helped to synthesize some substrates. T.X. wrote the paper with input from others. P. Zheng prepared the supplementary information.

\section{Competing interests}

The authors declare no competing interests.

\section{Additional information}

Supplementary information The online version contains supplementary material available at https://doi.org/10.1038/s41467-021-21947-1.

Correspondence and requests for materials should be addressed to T.X.

Peer review information Nature Communications thanks Jia-Rong Chen and Malcolm P. Huestis for their contribution to the peer review of this work.

Reprints and permission information is available at http://www.nature.com/reprints
Publisher's note Springer Nature remains neutral with regard to jurisdictional claims in published maps and institutional affiliations.

(c) (i) Open Access This article is licensed under a Creative Commons Attribution 4.0 International License, which permits use, sharing, adaptation, distribution and reproduction in any medium or format, as long as you give appropriate credit to the original author(s) and the source, provide a link to the Creative Commons license, and indicate if changes were made. The images or other third party material in this article are included in the article's Creative Commons license, unless indicated otherwise in a credit line to the material. If material is not included in the article's Creative Commons license and your intended use is not permitted by statutory regulation or exceeds the permitted use, you will need to obtain permission directly from the copyright holder. To view a copy of this license, visit http://creativecommons.org/ licenses/by/4.0/.

(C) The Author(s) 2021 